\title{
Repair of aortic arch coarctation and innominate artery aneurysm with branched Dacron graft
}

\author{
John S. Ikonomidis, MD, PhD, Peter Y. Kim, MD, and Fred A. Crawford, Jr, MD
}

A

ortic arch coarctations that persist into adult life are very rare. ${ }^{1-5}$ Aortic arch repairs requiring complete exclusion of aortic tissue have required separate implantation of the great vessels directly into the main graft or, more commonly, fabrication by the surgeon of a branched graft before the repair. The recent availability of prefabricated branched Dacron grafts greatly facilitates these unusual operations. We report a unique case of aortic arch coarctation and innominate artery aneurysm associated with severe mitral insufficiency that was successfully treated with branched Dacron graft arch repair and mitral annuloplasty.

\section{Clinical Summary}

A 54-year-old woman had been followed for a number of years with serial echocardiograms related to asymptomatic mitral insufficiency. The patient had a history of supraventricular tachycardia that was well controlled with medications until more recently, when she noticed increasing episodes of palpitations. In addition, the patient began to have effort dyspnea. Echocardiograms at this time showed evidence of moderate-to-severe mitral insufficiency (Figure 1, A), with no valvular or chordal abnormalities, left atrial enlargement, and left ventricular hypertrophy. The patient had no family history of coronary or aortic disease. Physical examination revealed asymmetric arm blood pressures, a grade III/VI diastolic murmur, and markedly diminished femoral pulses with absent lower extremity pulses.

The patient was referred for cardiac catheterization before mitral valve repair. At the time of cardiac catheterization, it was not possible to pass a guide wire across the aortic arch through a right femoral approach. The right brachial artery was then used to successfully access the ascending aorta. An aortogram revealed evidence of an aortic arch coarctation involving all 3 arch vessels (Figure 2, A) in association with an innominate artery aneurysm. The mean gradient across the coarctation was $44 \mathrm{~mm} \mathrm{Hg}$. There was no evidence of aortic valve insufficiency. The coronary arteries had minor luminal irregularities, and the left ventricular wall motion was normal, with evidence of moderate-to-severe mitral

\footnotetext{
From the Division of Cardiothoracic Surgery, Medical University of South Carolina, Charleston, SC.

Received for publication Sept 15, 2003; revised Oct 6, 2003; accepted for publication Oct 10, 2003.

Address for reprints: John S. Ikonomidis, MD, PhD, Cardiothoracic Surgery, Medical University of South Carolina, Suite 409 CSB, 96 Jonathan Lucas St, Charleston, SC 29464 (E-mail: ikonomij@musc.edu).

J Thorac Cardiovasc Surg 2004;127:579-81

$0022-5223 / \$ 30.00$

Copyright (C) 2004 by The American Association for Thoracic Surgery

doi:10.1016/j.jtcvs.2003.10.017
}
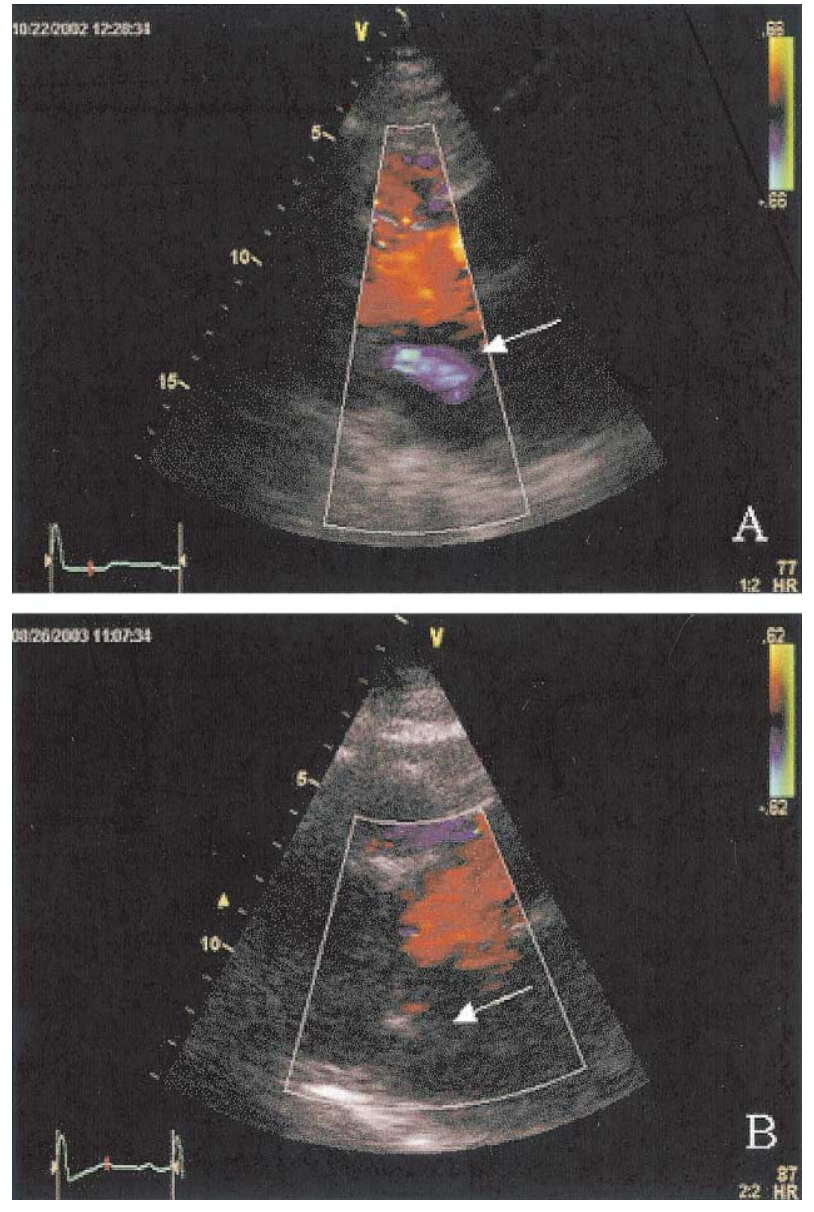

Figure 1. A, Preoperative transthoracic echocardiogram (parasternal long-axis view) showing centrally directed jet of mitral insufficiency (arrow) with color Doppler flow mapping. B, Postoperative image showing no mitral insufficiency (arrow).

insufficiency. It was recommended that this patient undergo aortic arch and innominate artery replacement in conjunction with mitral valve repair or replacement. On discussion of the risks and benefits of this procedure, the patient provided informed consent.

At the operation, a right infraclavicular incision was used to obtain proximal and distal control over approximately 2.5 to $3 \mathrm{~cm}$ of the axillary artery. A median sternotomy was then performed, and the skin incision was extended to the anterior right neck overlying the lower third of the right sternocleidomastoid muscle. The innominate vein was divided to obtain appropriate exposure of the aortic arch. Careful dissection was undertaken to expose the aortic arch coarctation and great vessels, including the aneurysmal innominate artery to its bifurcation. Both the left and right recur- 

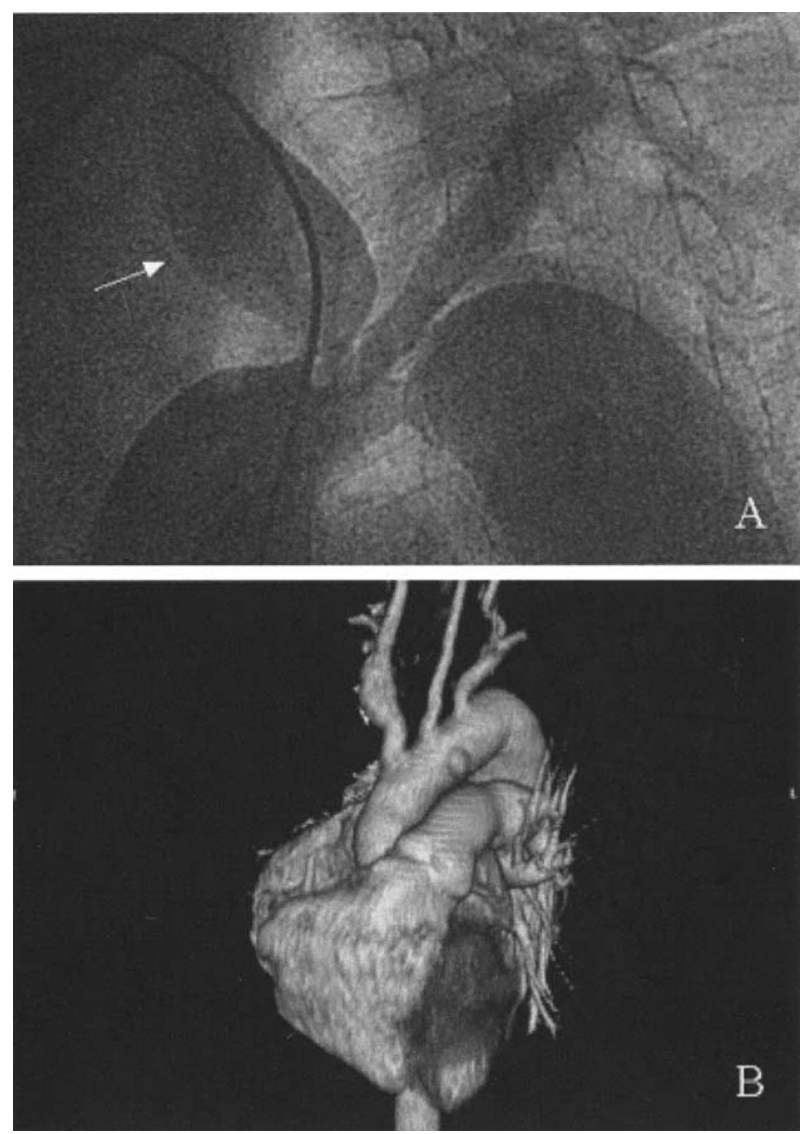

Figure 2. A, Preoperative aortogram showing arch coarctation and innominate artery aneurysm (arrow). B, Reformatted 3-dimensional reconstruction of postoperative computed tomographic scan showing intact aortic arch repair. The cannulation graft site is seen as a small out-pouching on the anterior surface of the graft.

rent laryngeal nerves were identified and preserved. The patient was systemically heparinized, and a $14 \mathrm{~F}$ arterial cannula (DLP; Medtronic, Minneapolis, Minn) was placed in the right axillary artery. A 7-mm, $65^{\circ}$, angled Soft Flow cannula (Terumo; Sarns, Ann Arbor, Mich) was placed in the ascending aorta just distal to the area of coarctation. Cardiopulmonary bypass and systemic cooling was commenced after bicaval venous cannulation through the right atrium and superior vena cava. Cardiac arrest was obtained by using cold antegrade blood cardioplegia and maintained with cold retrograde cardioplegia and a left ventricular cooling jacket.

A standard left atriotomy was performed posterior to the Sondergaard groove. Multiple attempts to position atrial retractors did not provide adequate visualization of the mitral valve. Therefore the superior vena cava was transected just cephalad to the sinoatrial node, and the atriotomy was extended across the dome of the left atrium posterior to the aorta and pulmonary artery. ${ }^{6-8}$ Excellent exposure of the mitral valve resulted, and careful examination revealed a morphologically normal valve and chordal apparatus. It was believed at this point that the mitral insufficiency was most likely caused by annular dilatation, and a 26-mm Cosgrove-Edwards annuloplasty band (model 4600; Edwards Lifesciences, Irvine, Calif) was placed without difficulty. Static testing of the valve at this point revealed no evidence of mitral insufficiency. The left atriotomy was closed, and the superior vena cava was reanastomosed.

At this point, the bladder temperature had reached $18^{\circ} \mathrm{C}$, and cooling had taken place for approximately 50 minutes. The pump was discontinued, and small vascular clamps were placed on the distal innominate artery, left common carotid artery, and left subclavian artery. The aortic arch cannula was clamped and removed, and selective antegrade cerebral perfusion was administered through the right axillary artery cannula at $18^{\circ} \mathrm{C}$ and a rate of $300 \mathrm{~mL} / \mathrm{min}$. The ascending aorta was transected, and the entire aortic arch with its narrowed segment was removed in addition to approximately two thirds of the innominate artery aneurysm. A 24-mm Dacron graft containing 4 separate Dacron side branches (Meadox; Boston Scientific, Natick, Mass) was used for the arch replacement. The anastomotic order proceeded from posterior to anterior, starting with the proximal descending thoracic aorta and followed by the left subclavian artery, after which the Soft Flow cannula was deaired and inserted into a side branch of the graft. The distal aspect of the arch repair was deaired, and a clamp was placed across the graft, allowing cold perfusion of the left subclavian artery and distal descending thoracic aorta at a cumulative rate of approximately $1 \mathrm{~L} / \mathrm{min}$. At this point, the left common carotid, innominate artery, and ascending aortic anastomoses were performed, with sequential crossclamp application across the main graft proximal to each newly constructed anastomosis. The aortic valve was a normal-appearing tricuspid structure. During rewarming, the axillary artery cannula was removed, and the artery was repaired transversely. The patient was weaned from cardiopulmonary bypass without difficulty. Intraoperative transesophageal echocardiography showed no mitral insufficiency. The total cardiopulmonary bypass time was 203 minutes, with a crossclamp time of 136 minutes and a perfusion-supplemented hypothermic circulatory arrest time of 42 minutes.

Pathology of the coarcted segment showed evidence of luminal stenosis consistent with coarctation and moderate arterial sclerosis. The patient had an uneventful postoperative course, with the exception of persistent left arm swelling, and was discharged home on postoperative day 7 .

The patient returned for follow-up at 1 and 10 months, at which time she felt well, with complete resolution of the left arm edema. A computed tomographic angiogram scan revealed an intact arch repair with no evidence of branch vessel anastomotic stenosis (Figure 2, B). In addition, transthoracic echocardiography showed no evidence of mitral insufficiency (Figure $1, B$ ).

\section{Discussion}

Although narrowing of the aortic arch is not infrequently seen in the setting of congenital heart surgery, aortic coarctations that persist into adult life are unusual, and those that originate between the arch vessels are very rare, constituting approximately $1 \%$ of all coarctation cases. ${ }^{4-8}$ Although aortic coarctation often occurs as an isolated malformation, it is also associated with a bicuspid valve in up to $85 \%$ of patients and also with other associated anomalies, including left heart obstructive lesions, congenital abnormalities of 
the mitral valve, ventricular septal defects, and patent ductus arteriosus. The pathogenesis of the stenosis is not well understood but seems to involve at least 2 mechanisms. The classic explanation suggests that insufficient cardiac output during gestation causes altered hemodynamics, which inhibit the normal growth of the fourth left aortic arch. ${ }^{9}$ Another study indicates that coarctation is characterized by the migration of smooth muscle cells from the intermediate to the subendothelial space, fragmentation of the internal elastic lamina, and subsequent separation of the endothelial cells from the internal elastic lamina. ${ }^{10}$ Although the exact genetic predisposition to this anomaly is unclear, an association has been made between the deletion of chromosome $22 \mathrm{q} 11$ and anomalies of the aortic arch and its branches. ${ }^{11}$

Prefabricated branched grafts greatly facilitate total aortic arch repairs. Separate reimplantation of the great vessels into the smaller side branches gives the surgeon flexibility to perform other procedures, such as resection of an innominate artery aneurysm, as described here. The small suture lines are less likely to bleed, and if hemorrhage does occur, it is easier to identify and control because the repair is more mobile and hence more accessible than a classic arch repair involving implantation of all 3 great vessels as a Carrel aortic island. The branched graft is particularly useful in cases in which complete exclusion of aortic tissue is desirable, such as in the present patient or in patients with established connective tissue abnormalities. Use of selective antegrade cerebral perfusion and sequential posterior to anterior clamping of the graft with distal perfusion reduces the amount of true cerebral ischemic time, thereby increasing the likelihood of successful neurologic recovery from a prolonged circulatory arrest period. ${ }^{5}$

In the present case the patient had a grossly normal aortic and mitral valve. The cause of the patient's mitral insufficiency was likely not related to the specific pathology of the aortic coarctation but rather might have been related to annular dilatation resulting from hypertension and persistent high afterload as a result of the coarctation. Although the insufficiency might have disappeared after coarctation repair alone, it was believed that the patient's symptoms and the degree of insufficiency merited mitral annuloplasty.

\section{References}

1. Winer HE, Kronzon I, Galssman E, Cunningham JN Jr, Madayag M. Pseudocoarctation and mid-arch aortic coarctation. Chest. 1977;72: 519-21.

2. Aris A, Subirana MT, Ferres P, Torner-Soler M. Repair of aortic coarctation in patients more than 50 years of age. Ann Thorac Surg. 1999;67:1376-9.

3. Katsumata T, Westaby S. Operation for mid-arch coarctation. Ann Thorac Surg. 1999;67:1386-90.

4. Corno AF, Botta U, Hurni M, Payot M, Sekarski N, Tozzi P, von Segesser LK. Surgery for aortic coarctation: a 30 years experience. Eur J Cardiothorac Surg. 2001;20:1202-6.

5. Rokkas CK, Murphy SF, Kouchoukos NT. Aortic coarctation in the adult: management of complications and coexisting arterial abnormalities with hypothermic cardiopulmonary bypass and circulatory arrest. J Thorac Cardiovasc Surg. 2002;124:155-61.

6. Selle JG. Temporary division of the superior vena cava for exceptional mitral valve exposure. J Thorac Cardiovasc Surg. 1984;88:302-4.

7. Barner HB. Combined superior and right lateral left atriotomy with division of the superior vena cava for exposure of the mitral valve. Ann Thorac Surg. 1985;40:365-7.

8. Kyger ER III, Coselli M, Kalchoff WP. A method for improved exposure of the mitral valve: cannulation of innominate vein and division of superior vena cava for extended left atriotomy in mitral valve operations. J Thorac Cardiovasc Surg. 1986;91:143-6.

9. Larsen WJ. Human embryology. 2nd ed. New York: Churchill Livingstone; 1997. p. 173-7.

10. Jimenez M, Daret D, Choussat A, Bonnet J. Immunohistological and ultrastructural analysis of the intimal thickening in coarctation of the human aorta. Cardiovasc Res. 1999;41:737-45.

11. McElhinney DB, Clark BJ III, Weinberg PM, Kenton ML, McDonaldMcGinn D, Driscoll DA, et al. Association of chromosome 22q11 deletion with isolated anomalies of aortic arch laterality and branching. J Am Coll Cardiol. 2001;37:2114-9. 